\title{
HIDATIDOSIS CEREBRAL PRIMARIA MÚLTIPLE: DESCRIPCIÓN DE UN CASO Y REVISIÓN DE LA LITERATURA
}

\section{Primary multiple cerebral hydatid disease: a case report and literature review}

\author{
Jean Marcel Castelo-Vega ${ }^{1, \mathrm{a}, \mathrm{E}}$, Sheyla Ponce Pérez ${ }^{2, \mathrm{~b}}$ \\ ${ }^{11}$ Servicio de Neurocirugía, Hospital Antonio Lorena, Universidad Andina del Cusco, Cusco, Perú, \\ ${ }^{2}$ Escuela de Medicina Humana, Universidad Andina del Cusco, Cusco, Perú \\ ${ }^{\mathrm{a}}$ Medico Neurocirujano \\ ${ }^{\mathrm{b}}$ Medico Cirujano \\ Đ'neuro_cast@yahoo.es
}

\begin{abstract}
Resumen
La hidatidosis cerebral múltiple es una infestación parasitaria potencialmente mortal causada por el estadio larvario del Echinococcus granulosus, siendo la mayoría de los quistes parenquimales de origen secundario; no obstante, la enfermedad primaria es extremadamente rara y su cuadro clínico se relaciona con el número, tamaño y ubicación de las lesiones. El diagnóstico requiere de imágenes cerebrales y el tratamiento es, casi siempre neuroquirúrgico, complementado con medicación antiparasitaria. Los autores presentamos una síntesis de los registros clínicos, imágenes, reporte quirúrgico e histopatología del caso de un paciente con hidatidosis cerebral primaria múltiple manejado en el Hospital Antonio Lorena del Cusco, en mérito a no haber sido documentado antes en el país y al éxito en su tratamiento especializado.

Palabras clave: hidatidosis cerebral múltiple, quiste parenquimal, neurocirugía.
\end{abstract}

\section{Abstract}

Multiple cerebral hydatidosis is a potentially deadly parasitic infestation caused by the larval stage of Echinococcus granulosus, with the majority of parenchymal cysts of secondary origin; however, the primary disease is extremely rare and its clinical picture is related to the number, size and location of the lesions. Diagnosis requires brain imaging and treatment is almost always neurosurgical, supplemented with antiparasitic medication. The authors present a synthesis of the clinical records, images, surgical report and histopathology of the case of a patient with multiple primary cerebral hydatidosis managed at the Antonio Lorena Hospital in Cusco, in recognition of not having been previously documented in the country and the success in your specialized treatment.

Keywords: multiple cerebral hydatid disease, parenchymal cyst, neurosurgery.

Citar como: Castelo-Vega, JM., Ponce, S. (2018). Hidatidosis cerebral primaria múltiple: descripción de un caso y revisión de la literatura. Rev Yachay, 7(1),505-510.

Recibido: 16-09-2018; Aceptado 07-12-2018

\section{Introducción}

La hidatidosis cerebral es una infestación parasitaria rara (1-2\%) y potencialmente mortal causada por el estadio larvario del Echinococcus granulosus, siendo la mayoría de los quistes intracraneales secundarios y únicos (90\%), más frecuentemente supratentoriales y en el territorio de la arteria cerebral media. Sin embargo, los quistes cerebrales múltiples y primarios son aún mucho más raros y se han descrito mayormente en niños y jóvenes, en quienes se ha relacionado el cuadro clínico con el número de quistes, su tamaño y ubicación en el parénquima encefálico. Luego del acercamiento diagnóstico más probable mediante neuroimágenes cerebrales —como tomografía espiral multicorte (TEM) o resonancia magnética (RM) - , la conducta terapéutica es, casi siempre, quirúrgica y se complementa con medicación antiparasitaria. 


\section{Presentación de caso clínico}

Presentamos la revisión nosológica de los registros clínicos, imagenológicos, quirúrgicos e histopatológicos de un paciente con hidatidosis cerebral múltiple primaria, que fuera manejado previamente en otra institución, ingresa por emergencia al Servicio de Neurocirugía del Hospital Antonio Lorena de Cusco, donde se realiza su evaluación y manejo especializado. La identidad del paciente ha sido plenamente resguardada.

Filiación y antecedentes. El caso corresponde a un paciente varón de 23 años, procedente de la ciudad de Sicuani en Cusco, de ocupación obrero y con grado de instrucción secundaria incompleta. Siempre presentó un estado de salud bueno, siendo el antecedente más destacable el contacto prolongado con un perro ovejero en su residencia habitual.

Anamnesis. Inició su enfermedad 9 meses antes de su ingreso a nuestro nosocomio, en forma insidiosa y con evolución progresiva, ha presentado cefalea frontal de tipo gravativa y opresiva y con intensidad creciente (hasta EVA 8/10) asociado secuencialmente con trastornos de la conducta y la memoria de trabajo, visión borrosa bilateral y de predominio izquierdo y, recientemente, náuseas y vómitos. Por tal razón, fue referido a un hospital de la ciudad de Cusco, donde recibió medicación con corticoides, manitol y fenitoína, indicándosele, además, su inmediata 'referencia a Lima', para manejo especializado. No obstante, ante las limitaciones o negativa del paciente para su traslado y debido a la falta de respuesta terapéutica en los meses posteriores, el paciente ingresa por emergencia del "Hospital Antonio Lorena de Cusco", buscando atención alternativa al presentar: deterioro neurológico por convulsiones generalizadas tipo 'gran mal' y vómitos explosivos.

Examen físico. En la evaluación general fue examinado en decúbito dorsal, despierto, con ventilación espontánea y signos vitales estables, así como un perfil cardíaco y pulmonar normal, pero era evidente una facies tipo cushingnoide. En la evaluación neurológica se hallaba orientado en tiempo, espacio y persona, con 15 puntos en la escala de Glasgow y signos de bradifrenia, moria e ideación impulsiva y paranoide (puntaje mini-mental state exam o MMSE de 24/30 y evaluación psicológica); mientras que un examen de su agudeza visual demostró en el lado izquierdo cuenta dedos a 5 m., y en el lado derecho 20/25, asociado a papiledema bilateral leve sin compromiso foveal, según el examen de fundoscopia indirecta (evaluación oftalmológica).

Pruebas auxiliares. Se le realizó una imagen de TEM (Tomografía Espiral Multicorte) cerebral contrastada, la que puso en evidencia signos de múltiples lesiones quísticas a nivel subcortical frontal izquierdo bien circunscritas, asociadas en conglomerado, de tamaños variables y con extensión hacia la profundidad de los ventrículos laterales, el cuerpo calloso y la cisterna silviana izquierda. Fueron evidentes los signos de hipertensión endocraneana, aunque sin poder verificarse signos de edema ni realce de contraste, en o alrededor de dichas lesiones (fig. 1).

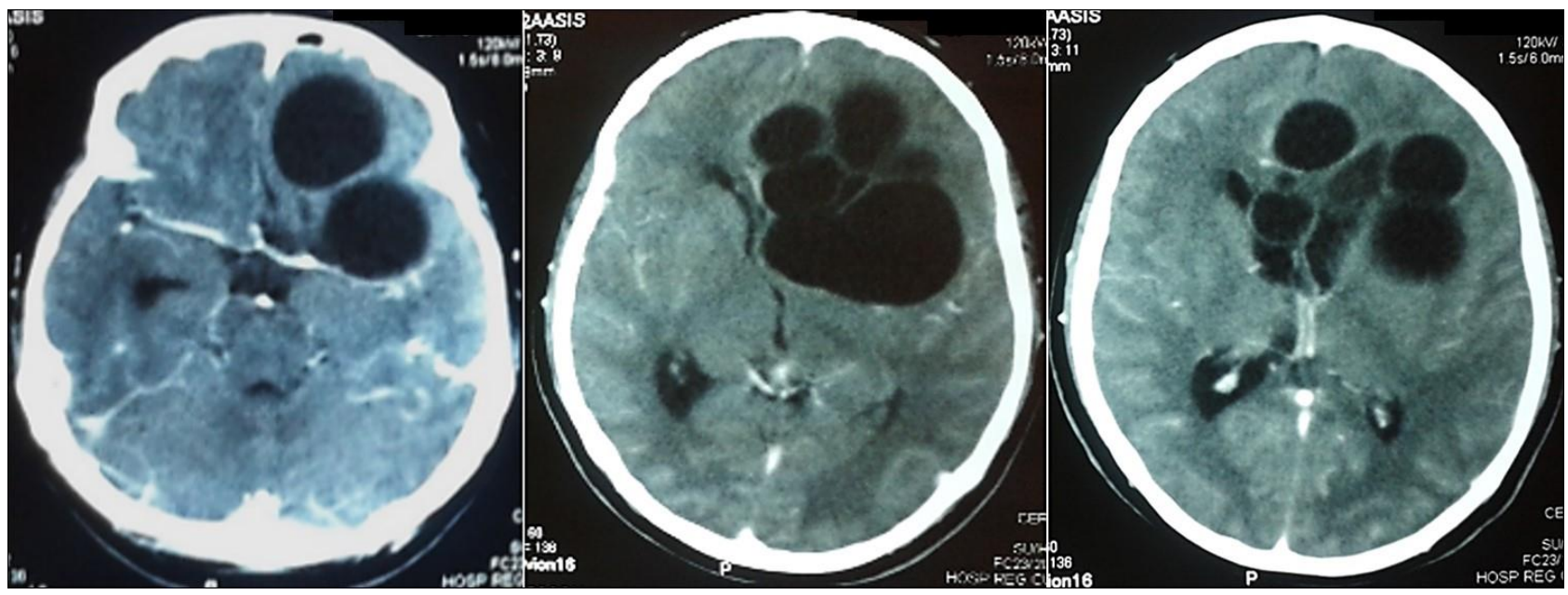

Fig. 1: Imagen secuencial de TEM cerebral axial y contrastada que demuestra la lesión multiquística en conglomerado, dispuesta a nivel subcortical frontal, cisternal y ventricular. Se advierte el efecto de masa importante y la ausencia de captación de contraste y de edema perilesional.

Rev. Yachay volumen (7) Número (1), enero-diciembre 2018 
Entre las pruebas de laboratorio destacaron: hematimetría con linfopenia (2-4\%), lámina periférica sin alteraciones, bioquímica con hipercortisolemia e hiperglicemia, e inmunología con prueba de Inmunoblot negativo para hidatidosis (Instituto Nacional de Salud) y, prueba de ELISA para VIH también negativa (Hospital Antonio Lorena). Y, entre las pruebas de imagen relevantes describimos: ecocardiografía transtorácica sin alteraciones estructurales (Servicio de Cardiología, Hospital Antonio Lorena) (fig. 2a), radiografía de tórax y ecografía abdominal sin alteraciones (Servicio de Diagnóstico por Imágenes, Hospital Antonio Lorena) (fig. 2b).
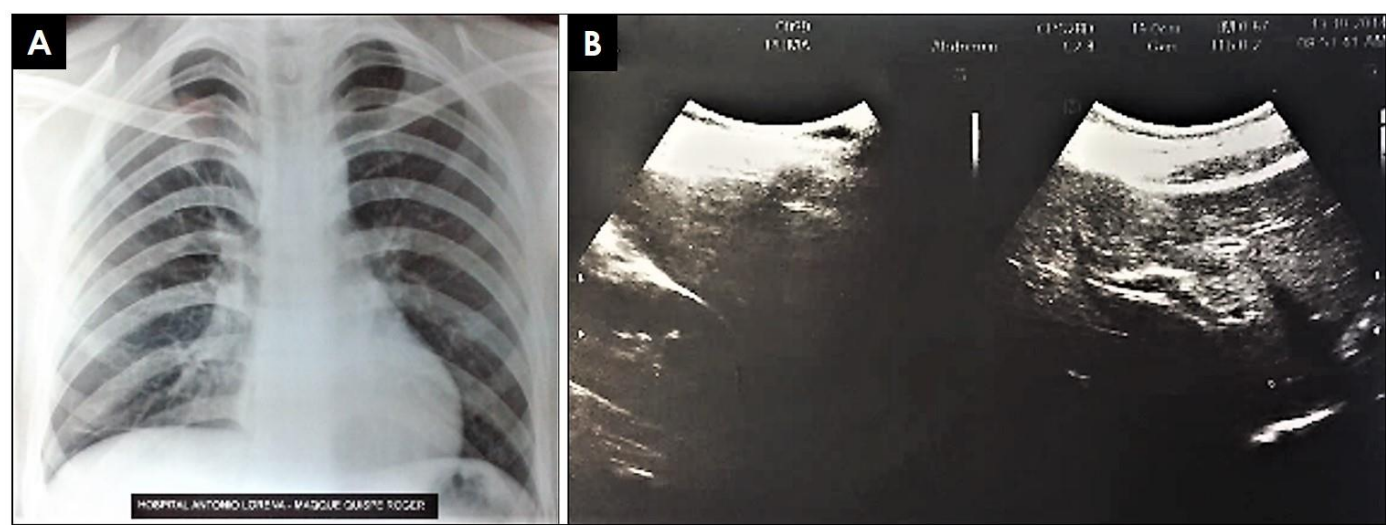

Fig. 2: Imágenes de radiografía de tórax $(\boldsymbol{A})$ y ecografía abdominal $(\boldsymbol{B})$ que no revelan signos de lesión quística en probable relación a hidatidosis pulmonar o hepática, respectivamente.

Tratamiento. Luego de una pronta evaluación por nuestro servicio, y previo consentimiento informado expreso de su familia, se decidió el manejo neuroquirúrgico de urgencia en virtud del avanzado deterioro neurológico y riesgos de muerte del paciente. El abordaje consistió en una craneotomía fronto-temporal izquierda, amplia; cortisectomía transulcal entre F2 y F3 izquierdos en su margen anterior y disección más exéresis de los quistes — según las técnicas de Dowling-Orlando (6 lesiones) -e irrigación-salinización con solución hipertónica al 7,5\% (3 lesiones) (fig. 3). La cirugía se realizó en un lapso de 3 h y 30 min, se estimó un sangrado de 190 ml., y no hubo complicaciones reportadas por anestesiología ni por nuestro equipo. Dejamos un drenaje tubular calibre $12 \mathrm{fr}$, en el lecho y se enviaron las muestras para estudio histopatológico.
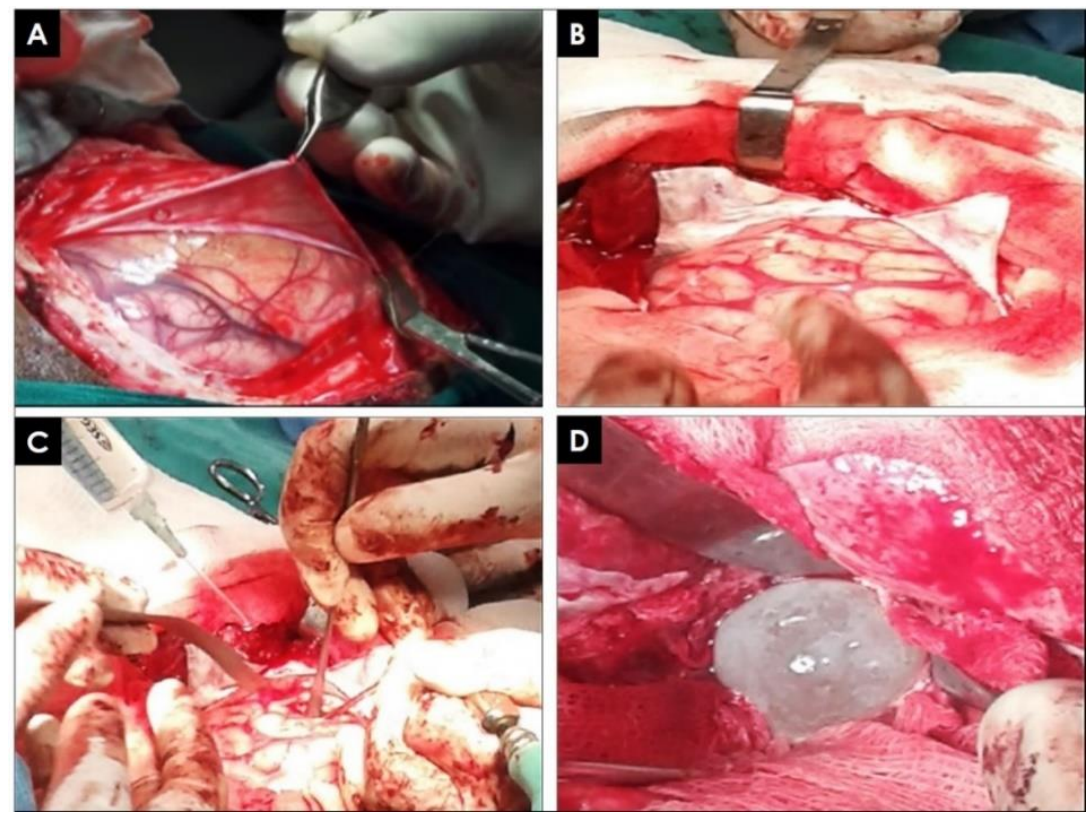

Fig. 3: Imágenes del acto quirúrgico. $\boldsymbol{A}$, craneotomía y durotomía; $\boldsymbol{B}$, exposición del parénquima congestivo y edematoso; $\boldsymbol{C}$, disección transulcal entre los giros F2 y F3 izquierdos; $\boldsymbol{D}$, quistectomía in-toto según técnica de Dowling-Orlando. 


\section{Resultados.}

Al otro día, el paciente se encontraba despierto (en Glasgow 15), sin déficit agregado; con la herida operatoria en buen estado y el drenaje con escaso contenido seroso. Se le realizó una TEM cerebral de control, la que demostró la exéresis completa de 9 de 10 quistes con uno pequeño residual de $1,5 \mathrm{~cm}$., en la profundidad del cuerpo calloso (fig. 4); no obstante, los signos de hipertensión endocraneana remitieron íntegramente, por lo cual, se retiraron gradual y decididamente los corticoides, comprobando la remisión de la linfopenia e hiperglicemia en los días posteriores.

El estudio histopatológico reportó formaciones quísticas y membranosas de diversos tamaños, con cutícula granulomatosa y numerosos escólices al interior (Laboratotio de Anatomía Patológica) (fig. 5).

El paciente fue dado de alta 10 días después, en buenas condiciones generales y neurológicas, siendo controlado ambulatoriamente hasta por 4 meses; logrando mantenerse asintomático y con mejoría apreciable en su perfil cognitivo (MMSE 28/30), así como en su agudeza visual (ojo izquierdo 20/40). En dicho periodo recibió tratamiento médico complementario con antiparasitarios cisticidas (albendazol $400 \mathrm{mg}$ por vía oral, c/8 horas, por 2 semanas y en 2 ciclos).

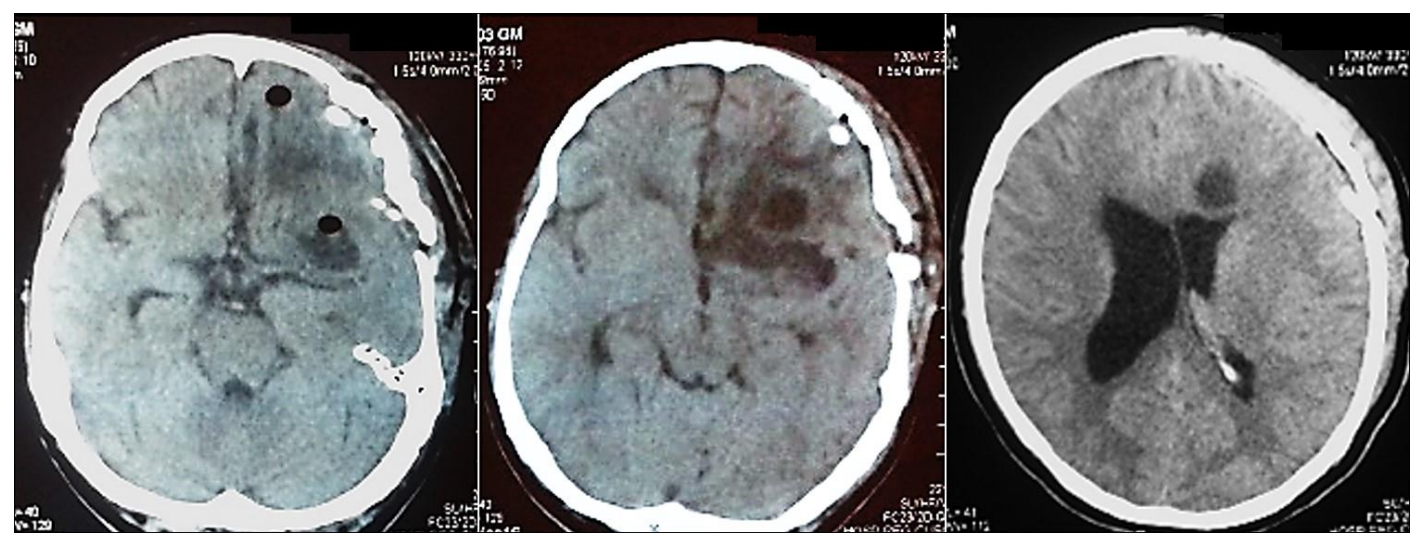

Fig. 4: Imagen secuencial de TEM cerebral axial simple y postoperatoria, que demuestra la exéresis total de $9 / 10$ quistes (izquierda y centro) y un pequeño residual de $1,5 \mathrm{~cm}$ (derecha). Es evidente la resolución de los signos de hipertensión endocraneana.

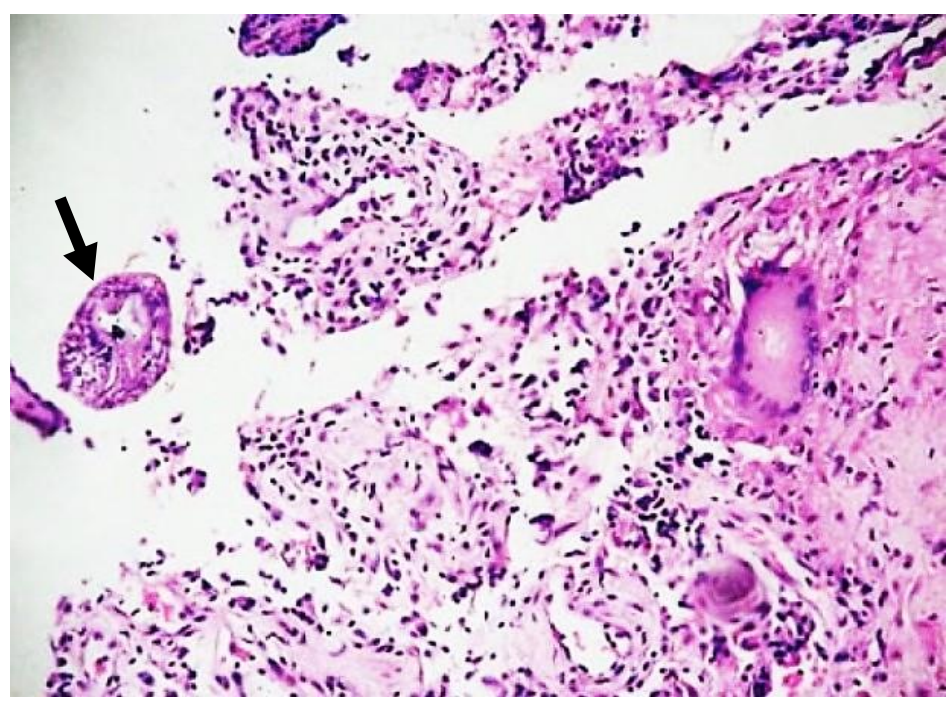

Fig. 5: Microfotografía del resultado histopatológico que muestra la membrana cuticular con intenso infiltrado inflamatorio polimorfonuclear y signos de necrosis. La flecha negra señala la estructura de un escólex hidatídico. 


\section{Discusión}

La hidatidosis cerebral primaria múltiple es una patología de presentación anecdótica (Satya Bhusan Senapati, Deepak Kumar Parida, A. S. Pattajoshi, Amiya Kumar Gouda, and Ashis Patnaik, 2015 ), habiéndose reportado solo 20 casos en el mundo en búsquedas relacionadas por PUBMED, SCOPUS, EBSCO, BIREME y Google Académico hasta la fecha (Meera M, Vrushali T, Tanaya, Krunal L., 2018). De hecho, no existe publicación conocida sobre un caso en el Perú. Resulta de la infestación directa de larvas de Echinococcus granulosus, sin la participación demostrable de otros órganos diana en los cuadros de hidatidosis clásicos como el pulmón y el hígado; quizás debido a la falta de un 'sistema inmune cerebral' efectivo que logre identificar y presentar a tiempo los antígenos parasitarios, a su sorprendente complacencia tisular cerebral al no generar reacción inflamatoria perilesional o, a la persistencia del conducto arterioso y el foramen oval en casos demostrados (Yaka U, Aras Y, Aydoseli A, Akcakaya MO, Sencer A, Imer M, Hepgul K., 2013). De hecho, muchas infestaciones parasitarias cerebrales, como la neurocisticercosis, desarrollan posibles mecanismos de mimetismo molecular o inmunológico que retrasan su detección oportuna y un eventual tratamiento menos agresivo.

La secuencia clínica descrita en la literatura relaciona los síndromes de irritación cortical (convulsiones motoras generalizadas), déficit focal neurológico o cognitivo (signos de disfunción lobar) e hipertensión endocraneana, según el número y ubicación de los quistes hidatídicos (Per H, Kumandaş S, Gümüş H, Kurtsoy A., 2009); en atención a lo cual, el caso presentado desarrolla similar conducta nosológica, aunque resulta mandatorio resaltar la concurrencia del factor epidemiológico por la procedencia del paciente, el cual es trascendente en el contexto geográfico de la ciudad de Sicuani y las zonas aledañas de la región del Cusco, donde existen focos endémicos bien identificados de enfermedad hidatídica.

Es normal que las pruebas de neuroimagen, como TEM o RM, revelen las lesiones en sospecha descritas como estructuras quísticas multiloculares o arracimadas y, con ausencia de reacción inflamatoria en la periferia (Erkutlu, I., Buyukhatipoglu, H., Alptekin, M., y Gok, A., 2008); hecho que también ha resultado característico en nuestro caso, donde la TEM cerebral demostró un conglomerado de lesiones quísticas con relación al diagnóstico hasta entonces inferido, aunque no pudimos completar el estudio de RM cerebral debido a la urgencia quirúrgica que demandaba el paciente. Si bien algunos autores resaltan que la caracterización imagenológica de las lesiones es un argumento sólido a favor del diagnóstico (Ravalji M, Kumar S, Shah A, Vaghela D, Makwana G, Singh K. CT and MRI, 2006), en nuestra experiencia, siempre consideramos a la neurocisticercosis como alternativa, sobre todo, en un contexto de endemicidad y elevada prevalencia en el Perú. Otros diagnósticos diferenciales pueden incluir quistes porencefálicos, quistes aracnoideos, infecciones (fúngicas, tuberculosas), abscesos y tumores quísticos cerebrales (por ejemplo, astrocitomas) (Bukte, Y., Kamanoglu, S., Nazaroglu, H., y cols, 2004).

Con relación a los estudios de ecocardiografía, radiografías de pulmón, ecografía abdominal o inmunología, los resultados son contradictorios o erráticos. De hallarse comunicaciones cardiacas derecha-izquierda, podríamos asumir la enfermedad como secundaria debido a la posibilidad de una metástasis parasitaria y su desarrollo posterior en el cerebro. Aunque no está probado que su presencia sea un factor de riesgo asociado en forma indiscutible (Meera, Modak; Vrushali, Thakar; Tanaya, Lahiri Krunal, 2018). Es así que, en nuestro caso, ninguna imagen extracerebral ha resultado útil para fortalecer el diagnóstico de un foco primario de hidatidosis, lo cual satisface la denominación de enfermedad cerebral primaria. Las pruebas inmunológicas — a diferencia de la neurocisticercosis-, suelen ser negativas con relación a la extensión de la enfermedad y, por ende, tampoco representan criterios diagnósticos válidos (Óp. Cit., 2009). Finalmente, no hay información relacionada a cambios en la hematimetría de los pacientes, por lo cual hemos asumido que la linfopenia tiene origen iatrogénico a causa de los corticoides.

El tratamiento de elección en la hidatidosis cerebral primaria múltiple es —indiscutiblemente- quirúrgico (Óp., Cit., 2013), sin opción de alternativas médicas, debiendo efectivizarse en cuanto sea inmediatamente posible por el estadio avanzado en el que se demuestra la sospecha diagnóstica (Cavuşoğlu H, Tuncer C, Ozdilmaç A, Aydin Y., 2009). Como acontece en el quiste hidatídico solitario, la técnica de elección es la exéresis descrita por Dowling y Orlando (o "parto del quiste") procurando la exéresis in toto del quiste, con escasa manipulación e hidrodisección periquística, buscando evitar su ruptura, la potencial diseminación de los escólices y las posibles reacciones anafilácticas subsecuentes (Izci Y, Tüzün Y, Seçer HI, Gönül E., 2008).

En el presente caso logramos retirar 6 quistes en forma completa, otros 3 se rompieron en el intento debido a la gran tensión superficial y fragilidad de sus paredes, que permite la conglutinación de lesiones; hecho que obligó a una inmediata y enérgica irrigación del lecho con solución salina hipertónica (7,5\%) en el intento de mitigar los potenciales efectos arriba descritos. No se verificaron reacciones adversas durante la cirugía, ni en los días o semanas posteriores al evento. 
El diagnóstico definitivo se establece por la confirmación histopatológica de las lesiones hidatídicas, complementando el tratamiento, según la mayoría de los autores, con esquemas de antiparasitarios específicos individualizados de acuerdo al caso (Óp. Cit Senapati y cols., 2015; Óp. Cit. Yaka y cols., 2013).

El medicamento más utilizado es el albendazol por su tolerancia y relativa efectividad; aunque, por cierto, no existen reportes conocidos sobre la duración y dosis más adecuadas en hidatidosis cerebral múltiple; con relación a lo cual debemos precisar que luego de 4 meses de seguimiento clínico o tomográfico, no evidenciamos signos de recurrencia de la enfermedad, pero sí notable disminución de las dimensiones del pequeño quiste residual en el control más reciente.

\section{Conclusiones}

La hidatidosis cerebral primaria múltiple es una enfermedad de origen parasitario y de presentación extremadamente infrecuente, cuya fisiopatología es aún poco conocida en atención a su conducta biológica e inmunológica, pero que requiere un elevado índice de sospecha clínico-epidemiológico y una evidencia de neuroimagen consistente para el acercamiento diagnóstico. La resección quirúrgica de los quistes es, sin duda, el tratamiento de elección; el cual realizado en forma oportuna y eficaz permite limitar el daño neurológico, de este modo, mejorar la calidad de vida del paciente. Aún es preciso reunir más información relacionada a su nosología, tanto como fortalecer las estrategias de control epidemiológico con fines de prevención primaria en el ámbito de nuestra región y el país.

\section{Referencias bibliográficas}

Bukte, Y., Kamanoglu, S., Nazaroglu, H., y cols. (2004). Cerebral hydatid disease: CT and MR imaging findings. Swiss Med Wkly, 134(7), $459-467$.

Cavuşoğlu H, Tuncer C, Ozdilmaç A, Aydin Y. (19 de abril de 2009). Multiple intracranial hydatid cysts in a boy. Turk Neurosurg, 7(2), $203-207$. Obtenido de https://www.ncbi.nlm.nih.gov/pubmed/19431138

Erkutlu, I., Buyukhatipoglu, H., Alptekin, M., y Gok, A. (15 de febrero de 2008). Where is the brain paranchyme?: "Primary cerebral multiple cyst hydatid". Intern Med, 47(4), 333. Obtenido de https://www.ncbi.nlm.nih.gov/pubmed/18277045

Izci Y, Tüzün Y, Seçer HI, Gönül E. (24 de junio de 2008). Cerebral hydatid cysts: technique and pitfalls of surgical management. Neurosurg Focus, 6. doi:10.3171/FOC/2008/24/6/E15.

Meera M, Vrushali T, Tanaya, Krunal L. (28 de mayo de 2018). Primary multiple intraventricular hydatid cysts in a child. (Epub, Ed.) PubMed, 8(1), 47-49. doi:10.4103/tp.TP_31_16

Meera, Modak; Vrushali, Thakar; Tanaya, Lahiri Krunal. (19 de diciembre de 2018). Primary multiple intraventricular hydatid cysts in a child. Tropical Parasitology, 47-49. Obtenido de http://www.tropicalparasitology.org/text.asp?2018/8/1/47/233331

Per H, Kumandaş S, Gümüş H, Kurtsoy A. (16 de marzo de 2009). Primary soliter and multiple intracranial cyst hydatid disease: report of five cases. (PubMed, Ed.) PubMed, 31(3), 228-233. doi: 10.1016/j.braindev.2008.03.009

Ravalji M, Kumar S, Shah A, Vaghela D, Makwana G, Singh K. CT and MRI. (2006). Features of the typical and atypical intracranial hydatid cysts: report of five cases. Indian Journal of Radiology and Imaging, 16(4), 727-723.

Satya Bhusan Senapati, Deepak Kumar Parida, A. S. Pattajoshi, Amiya Kumar Gouda, and Ashis Patnaik. (Apr-Jun de 2015 ). Primary hydatid cyst of brain: Two cases report. (W. Kluwer, Ed.) Asian Journal of Neurosurgery, 10(2), 175-176. doi:10.4103/1793-5482.152109

Yaka U, Aras Y, Aydoseli A, Akcakaya MO, Sencer A, Imer M, Hepgul K. (2013). Primary multiple cerebral hydatid disease: still symptomatic despite pathologically confirmed death of the cyst. PubMed, 23(4), 505-508. doi:10.5137/1019-5149.JTN.5826-12.1 